\title{
Clinical Aspects of Overlap Syndrome - Case Report and Literature Review
}

\section{Bogna Grygiel-Górniak*, Oscar Nicholas Godtfredsen, Gunnar Nyborg Eid, Nicholas Werczak, Mariusz Puszczewicz}

Department of Rheumatology and Internal Medicine, Poznan University of Medical Sciences, Poznan, Poland

*Corresponding Author: Bogna Grygiel-Górniak, Department of Rheumatology and Internal Medicine, Poznan University of Medical Sciences, Poznan, Poland, E-mail: bgrygiel@ump.edu.pl

Received: 04 May 2018; Accepted: 08 May 2018; Published: 10 May 2018

\begin{abstract}
We report a patient with overlap syndrome (systemic sclerosis ( $\mathrm{SSc}$ ) and polymyositis (PM)). The heterogeneous nature of systemic sclerosis may lead to a great diversity in the clinical presentation of the disease. With this case report we aim to demonstrate clinical manifestations of systemic sclerosis and polymyositis in an overlap-syndrome, with support from antibody profile and laboratory data.
\end{abstract}

Keywords: Overlap syndrome; Systemic sclerosis; Polymyositis; Pulmonary fibrosis; Treatment

\section{Case Report}

A 51-year old female patient came to the Pulmonology Ward in 2015 complaining of shortness of breath and reduced exercise tolerance for approximately 1 year. Clinical examination revealed a rash on the neck that spread throughout the upper part of thorax, in addition to swollen, reddish fingers with skin stiffness on the hands. The patient also suffered from a swollen face and swollen eyelids. On auscultation, crackles were heard in the lower lung fields. On X-ray, changes in the interstitium were seen, and computer tomography confirmed fibrotic areas located peripherally in the lower lobes. In HRCT (high definition computer tomography) fibrosis was found in both lungs peripherally in the lower posterior lobes. The most exacerbated patches of fibrosis were present in the eighth segment of the lung and the lung uvula. These changes drew upwards in the distal segments of the subsegmental bronchi and the diaphragm. Small lymph nodes were present in the aortopulmonary window, in the front of the mediastinum, in recesses and under the carina (Figure 1). However, tests of respiratory functions were normal. An echocardiography revealed some features, which suggest pulmonary arterial hypertension (PAH), but a right heart 
catheterisation did not confirm the PAH. Diffusing capacity of lung carbon monoxide (DLCO) was measured, with a result of $6.47 \mathrm{mmol} / \mathrm{min} / \mathrm{KPa}$.

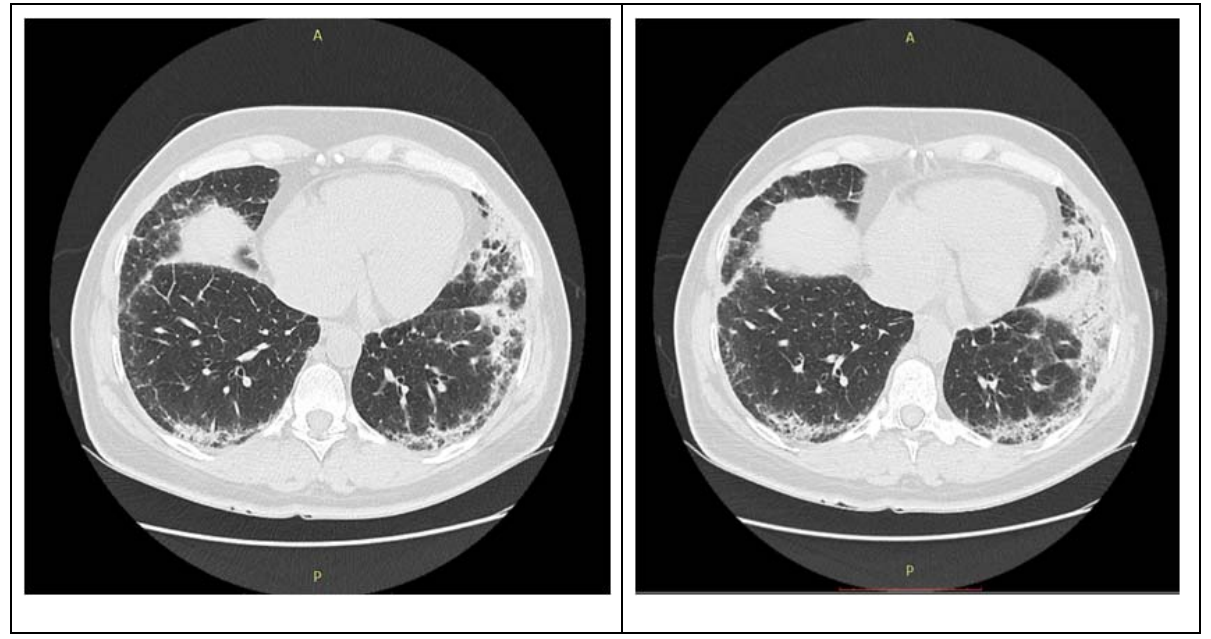

Figure 1: Chest HRCT showing bilateral reticular abnormalities and honeycombing with peripheral distribution in lower lobes.

A month later, the patient was hospitalized in the Thoracic Surgery Department, where a right-sided Video Assisted Thoracoscopic surgery was performed. A wedge resection of the lower lobe was done, and the surgery was uneventful. Laboratory tests revealed a serological ANA of 1:5120 with the presence of antibodies PM-Scl-100 and PM-Scl-75. Based on the clinical features and laboratory findings, a diagnosis of systemic scleroderma (SSc) was given. The patient was treated with pulses of methylprednisolone with cyclophosphamide given intravenously.

One year later, the patient was admitted to the Rheumatology Department with dyspnea on exertion and muscle weakness. A physical examination revealed redness, swelling and stiffness of the hands and face (modified Rodnan skin scoring - MRSS of 9 points). Besides this, a single telangiectasia on the face, microstomia (decreased mouth opening), microhelia (radial wrinkles around the mouth), painful muscles during palpation and muscle weakness of upper and lower extremities (3/4 points on the Lovet scale) were found. Crackles were heard at the base of the lung on both sides. Laboratory studies revealed increased concentrations of creatine kinase (CK), aldolase, LDH, ALT, AST, hypocholesterolemia, and hypertriglyceridemia. A nailfold videocapillaroscopy (NVC) revealed an acute SSc pattern with changes characteristic also for polymyositis (PM) (Figure 2).

After three cycles of treatment with methylprednisolone and cyclophosphamide given intravenously, the patient reported improved clinical signs (less severe exertional dyspnea) and an increased muscle strength in the lower limbs. In laboratory studies, diminished levels of creatine kinase and LDH were observed. The total dose of cyclophosphamide was $3600 \mathrm{mg}$ and the patient is now coming every month for consecutive infusions of 
methylprednisolone and cyclophosphamide intravenous infusion. Because of increased levels of LDL and triglycerides and a dietary intervention having no effect, a statin was introduced.

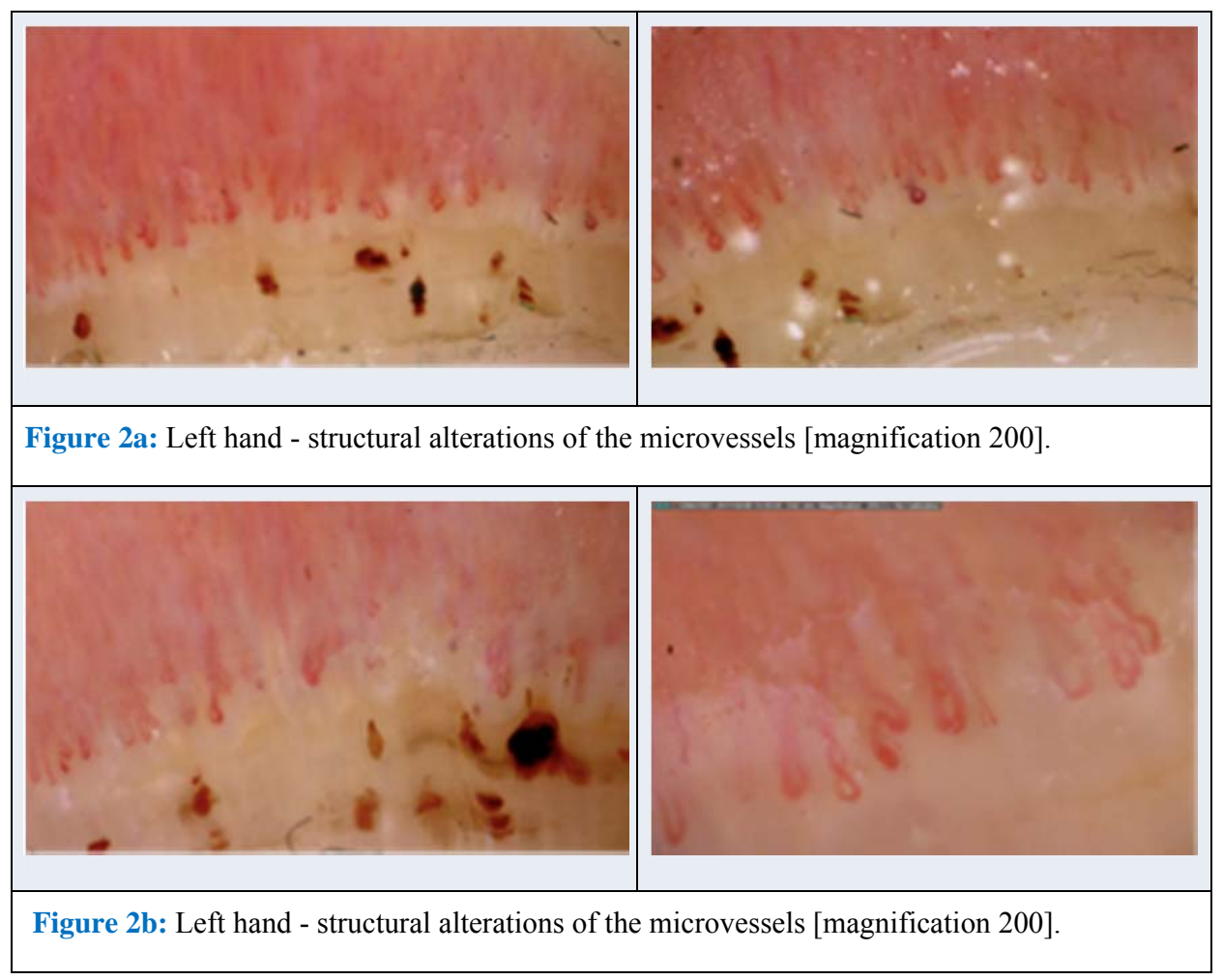

Figure 2: Nailfold videocapillaroscopy (NVC) acute SSc pattern. (magnification 200×).

\section{Discussion with A Review of the Literature}

In this study, characteristic skin, muscle and organ changes as well as characteristic laboratory and imaging data allowed us to diagnose systemic sclerosis ( $\mathrm{SSc}$ ) with polymyositis (PM). Initially only skin stiffness was present, while muscle involvement presented 12 months later. Typical skin changes (stiffness of the hands and face, including microstomia, microhelia and telangiectasia) are often observed in the course of SSc [1]. The skin is characterized by vascular obliteration and fibrosis of the skin, giving it a firm and taut character [2,3]. The scale used in the assessment of skin involvement is the modified Rodnan skin score (MRSS). Skin thickness is assessed at 17 different anatomical locations by palpation, and is then rated on a scale from 0-3 (where 0 represents normal skin, 1- mild, 2 - moderate, and 3 -severe skin thickening) [4]. Total amount of individual scores from each location is correlated with disease progression over time $[4,5]$. In this case, the MRSS was low (9 points), which indicates nonintensive skin thickening.

Patients with systemic sclerosis are classified into limited cutaneous (lcSSc) or diffuse cutaneous (dcSSc) type largely based on the distribution of the skin involvement, and this classification also has implications for treatment and prognosis. Patients with lcSSc are characterized by skin thickening limited to the digits (called sclerodactyly), 
which can expand to hands and forearms [1,2]. It is associated with a more benign course than the diffuse type, with organ involvement occurring only late in the disease $[2,3]$. The type of dcSSc has skin sclerosis extending proximal to the wrists, characteristically to the trunk and proximal limbs [3]. It is characterized by skin thickening developing over the first 3-12 months [2] and severe internal organ involvement early in the disease, leading to a high mortality rate [3].

In this case, the patient had a clinically apparent decrease in muscle function with elevated levels of creatine kinase and aldolase. The elevated muscle enzymes and clinically evident muscle involvement (weakness estimated using the Lovet scale) as well as PM-Scl antibodies enabled us to diagnose an overlap syndrome consisting of SSc with an inflammatory myopathy. Increased creatine kinase and aldolase levels in the clinical picture of systemic sclerosis is strongly suggestive of muscle involvement [6]; however, the absence of increased creatine kinase and aldolase levels does not exclude myositis [7]. There is no general consensus of how to classify the different types of myopathies associated with systemic sclerosis, as they can be analyzed based on histological findings, degree of clinical severity, response to corticosteroid therapy, electromyographic (EMG) patterns or MRI findings [6, 7]. Skeletal muscle involvement influences the quality of life and debilitates daily functioning [8]. However, muscle weakness without cardiac muscle changes has not been shown to increase mortality rate [9]. Conversely, muscle involvement in SSc is associated with a higher risk of myocardial complications [10], and an involvement of the heart would lead to a dramatic increase in 5 year mortality rates of up to $70 \%$ [11].

In SSc, the course of disease may be predicted when evaluating the specific antibody profile. Several antibodies has been linked to a certain type of disease progression. The more general antibodies that suggests SSc are mainly antitopoisomerase - anti-Scl-70 or ATA; anti-centromere antibodies and anti-RNA polymerase III antibody - ARA [5, 12]. Our patient was found to be ANA positive with a titer of 1:5120 (the patterns of nuclear staining was patchy and nucleolar), which is positive in up to $88 \%$ of patients with scleroderma [12]. The systemic sclerosis antibody profile also revealed the presence of the antibodies anti-Scl-70 and anti-PM-Scl. The anti Scl-70 autoantibody, also called the anti-topoisomerase 1-autoantibody after the type 1 topoisomerase target [13], is highly characteristic for SSc. The specificity of this marker is over $98 \%$ but the sensitivity is generally low (26-28\%) [5]. In addition, it has been observed that anti-topoisomerase 1 antibody is associated with severe pulmonary fibrosis $[14,15]$.

Other antibodies which have been detected in this patient are the anti-PM-Scl antibodies. These antibodies are found in polymyositis, dermatomyositis and systemic sclerosis, usually in overlap syndromes with varying predominating symptoms [16-18]. They are present only in 3\% of patients with SSc and up to $25 \%$ in patients with overlap syndromes (SSc with PM or Ssc with dermatomyositis - DM) [16, 19]. The anti-PM-Scl antibodies can be further subdivided into two major subgroups, the anti-PM-Scl-75 [20] and the anti-PM-Scl-100 [21]. Anti-PM-Scl-75 antibodies are more common in patients with dcSSc and are associated with a higher prevalence of lung involvement, joint contractures and muscular atrophy than anti-PM-Scl-75 negative patients. Besides, anti-PM-Scl75 antibodies reveal greater association with digital ulcers [22]. The anti-PM-Scl-100 positive patients have a higher frequency of elevated CK, and are less likely to suffer from gastrointestinal symptoms associated with SSc such as 
diarrhea, regular emesis or constipation [22]. The most common symptoms reported by patients with the anti-PMScl antibody are muscle weakness, Raynaud's phenomenon and arthritis [23].

Nailfold capillaroscopy (NFC) is a helpful method used in the diagnosis of SSc and later in the course of the disease as an indicator of disease activity $[24,25]$. This method is a non-invasive and useful technique used in the detection and follow-up of autoimmune rheumatic diseases [24]. Usually this method is used to differentiate primary Raynaud's phenomenon from secondary Raynaud's phenomenon [25]. In this case, NFC has been done to analyze the vascular pattern of the patient. We found a disorganized distribution of the normal distribution of capillaries, twisted enlarged capillaries and capillary hemorrhages (extravasates), enlargement of capillary loops, and 'budding' ('bushy') capillaries. Such changes are observed in systemic sclerosis as well as in polymyositis [26]. NFC is one of the examinations recommended as a part of the screening and monitoring of progression in SSc [27], and such a regiment has also been planned in this patient.

In this case, the patient reported a decreased exercise tolerance with dyspnea and chest pain a year before imaging studies. Factors affecting the outcome of an exercise tolerance test in patients with SSc and ILD needs further investigation. However, early identification of pulmonary involvement is essential, especially considering how insidious and heterogenous SSc-ILD can be. When a patient suspected of SSc presents with pulmonary findings on examination, several measures can be done to investigate the pulmonary involvement. One of them includes the pulmonary function tests (PFT), which on spirometry usually demonstrates a restrictive pattern representing the parenchymal involvement. Changes in the pulmonary function test can occur even before onset of significant clinical symptoms, therefore it should be frequently used as a screening measure [39]. In our patient, spirometry revealed a FEV1 of $85 \%$ and the Tiffeneau test (the ratio of FEV\% to FVC) was within normal ranges (values lower that $70 \%$ suggest obstruction). FVC is found to be reduced in $40-75 \%$ of patients with SSc [40]. There is no clear correlation between a decreased FVC (which is the gold standard test to evaluate ILD) and a decreased exercise tolerance test [41]. The pathophysiologic background of a reduced exercise tolerance test in patients with SSc and ILD is thought to be multifactorial, with a constellation of variables such as reduced oxygen diffusion across the alveolar membrane [42], vasodilatory failure in response to exercise [49] and diagnosed PAH [51]. However, simple measurement such as the 6 minute walk test (6MWT) is a reliable prognostic factor for patients with SSc and PAH, but not in patients with SSc and concomitant PAH and ILD [34]. The 6MWT is a viable test as a marker of disability in patients with SSc-ILD. Although nonspecific, it might be used to monitor endurance and stamina as a general indicator of disease progression [35]. However, some authors considered that this test has limited use. Many variables can influence the results, ranging from muscle function, pulmonary function, neuromuscular junction functioning to technician skill and patient motivation [36]. The 6MWT of this patients was within the recommended values [37].

The screening spirometry should include diffusing lung capacity of carbon monoxide (DLCO), a parameter shown to have a negative correlation with parenchymal inflammation which results in thickening of the interstitium and poor diffusion capacity. In fact, a decreasing DLCO has been shown to be the single most important marker for poor 
outcome, which elucidates disease progression [38]. Fortunately, in this case, the DLCO was only slightly decreased (lower than $80 \%$ ).

In this patient, evident changes of interstitial lung disease (ILD) were present (Figure 1). The occurrence of ILD in the course of systemic sclerosis (SSc) is fairly common, with evidence of interstitial changes in the lungs being reported in up to $91 \%$ of patients with scleroderma who had undergone a HRCT [39]. Degree of fibrosis detected on HRCT has been proven to predict future forced vital capacity (FVC\%) decline in the absence of effective treatment [40]. ILD secondary to systemic sclerosis is throughout literature regarded as the leading cause of morbidity and mortality in SSc. During the last 30 years a paradigm change has occurred regarding the leading cause of death in SSc. Previously, SRC (scleroderma renal crisis) was the leading cause of death in patients with SSc. However, now as SRC is effectively treated with ACE-I, pulmonary fibrosis has emerged as the leading cause of mortality with pulmonary arterial hypertension (PAH) coming second [41].

Pulmonary involvement in systemic sclerosis can be divided into two subtypes, SSc-ILD and PAH. There are some evidence showing that the specific subtype of pulmonary involvement can be linked with subtypes of systemic sclerosis. Pulmonary fibrosis were found to be more common in patients suffering from dcSSc with a prevalence of $53.4 \%$ compared to lcSSc with 34, 7\%. On the other hand, isolated PAH (in the absence of lung fibrosis) was found in $45 \%$ of patients with lcSSc and only $26 \%$ of patients with dcSSc [42]. Interestingly, pulmonary disease may even occur in the absence of skin involvement as an entity known as scleroderma sine scleroderma (ssSSc). In such cases idiopathic ILD is usually diagnosed; however, the presence of Raynaud's phenomenon, telangiectasias and positive antinuclear antibodies supports the possibility of ssSSc [49]. It has to be pointed out that SSc-ILD is a very heterogeneous disease. It may affect any aspect of the respiratory tract, and it may present itself at any time during the course of systemic sclerosis [51]. Occasionally, ILD may even be the initial manifestation of SSc, but in the course of the disease mucocutaneous telangiectasia, Raynaud's phenomenon and abnormal nailfold capillaries may be observed $[43,44])$.

It is of importance that the concurrent appearance of PAH with evidence of pulmonary fibrosis is common in SSc and overlap syndrome associated with SSc. In a study conducted by Demir et al., the reported incidence of PAH in SSc patients were reported in $24.1 \%$ patients; however only one-fifth from this group had only PAH, while the rest of patients had PAH associated with ILD [48]. Data was reported by Lefèvre et al., who found that the pooled 3 year survival rates in patients with SSc and only PAH were 59\%, while the same survival rate in SSc patients and concomitant PAH and ILD were only 35\% [48]. Authors of this review pointed that a greater score in the NYHA classification were associated with a worse prognosis in patients with SSc and only PAH. Nevertheless, NYHA classification were not a prognostic factor in patients with SSc associated with pulmonary hypertension and ILD [48].

In conclusion, patients with SSc with associated PAH and ILD are characterized by a higher mortality rate than patients with SSc or single PAH [34,45]. 
In this case, HRCT showed bilateral reticular abnormalities and honeycombing with peripheral distribution in lower lobes. HRCT is an essential part of routine evaluation of SSc-ILD. Abnormalities on HRCT was found in 55-65\% of all patients with SSc, and as high as $96 \%$ in patients with abnormalities on PFT. SSc-ILD presents typically on HRCT as ground-glass opacities (GGO) mixed with a pattern of pulmonary fibrosis, compatible with the nonspecific interstitial pneumonia (NSIP) pattern [49]. However, 11-37\% of patients with SSc-ILD was reported to have honeycomb cystic changes, which is a pattern more consistent with usual interstitial pneumonia (UIP) [51], so overlapping of the patterns may be seen.

One might argue that HRCT should be done as soon as possible after an initial diagnosis of SSC, even in the absence of pulmonary symptoms. As previously mentioned, SSc-ILD may be present on HRCT long before clinical symptoms. In a serial study done on 90 patients with SSc, 40 was found to be clear of pulmonary fibrosis on the initial scan, while 35 (88\%) continued to be free of pulmonary fibrosis on HRCT on follow-up for an average of five years [48]. HRCT may be used as a predictor of long-term prognosis with regard to SSc-ILD.

Other diagnostic tools worth mentioning are bronchoalveolar lavage fluid (BAL-fluid). BAL-fluid analysis has been an issue of debate. Even though the procedure is relatively safe, the findings seem to be nonspecific. Nonetheless, significant BAL lymphocytosis or eosinophilia may provide good support for a clinical diagnosis when combined with other diagnostic and clinical findings [49]. In 2012, The Official American Thoracic Society Practice Guideline released a report concluding that a BAL-fluid analysis frequently provides clinically useful information when the BAL-fluid is gathered under guidance from a HRCT, and the data obtained is used in conjunction with clinical information [51].

Echocardiography revealed a low TAPSE value and a thickened free wall of the right ventricle. This led to a suspicion of pulmonary hypertension (Table 1). Thus, the patient was admitted to the department of cardiac surgery, where a right heart catheterization (RHC) was performed. A slightly elevated systolic right ventricular pressure (RVP) was observed; however, mean pulmonary arterial pressure (mPAP), cardiac index (CI) and pulmonary vascular resistance (PVR) were within recommended values.

\begin{tabular}{|c|c|c|}
\hline & Patient Result & Normal Range \\
\hline \multicolumn{3}{|c|}{ Biochemical Analysis } \\
\hline $\mathrm{WBC}\left[10^{3} / \mu \mathrm{L}\right]$ & 9.4 & $4.0-10.4$ \\
\hline HGB $[\mathrm{g} / \mathrm{dL}]$ & 14.3 & $11.6-14.9$ \\
\hline $\operatorname{PLT}\left[10^{6} / \mu \mathrm{L}\right]$ & 4.82 & $3.78-5.5$ \\
\hline ESR $[\mathrm{mm} / \mathrm{h}]$ & 14 & $3-15$ \\
\hline ALAT $[\mathrm{U} / \mathrm{L}]$ & 29.1 & $9.0-36.0$ \\
\hline AST [U/L] & 32.0 & $10.0-31.0$ \\
\hline $\mathrm{CK}[\mathrm{U} / \mathrm{L}]$ & 155.2 & $26.0-140.0$ \\
\hline
\end{tabular}




\begin{tabular}{|c|c|c|}
\hline $\mathrm{LDH}[\mathrm{U} / \mathrm{L}]$ & 690.0 & $207.0-414.0$ \\
\hline ANA amount & 1/5120; & Norm $<1 / 80$ \\
\hline ANA profile & PM-Scl-75 and PM-Scl-100 & Negative \\
\hline Quantiferon Gold test & Negative & Negative \\
\hline \multicolumn{3}{|l|}{ Echocardiography } \\
\hline Mitral valve & \multicolumn{2}{|c|}{ morphologically normal, slight feedback wave } \\
\hline Tricuspid valve & \multicolumn{2}{|c|}{ morphologically normal, slight feedback wave } \\
\hline Left ventricular contraction & \multicolumn{2}{|c|}{ without segmental ventricular contraction. right ventricle with thickened free wall } \\
\hline $\mathrm{EF} \%$ & \multicolumn{2}{|l|}{$60 \%$} \\
\hline TAPSE & \multicolumn{2}{|l|}{$2.6 \mathrm{~cm}($ norm $>15 \mathrm{~mm})$} \\
\hline RVSP & \multicolumn{2}{|l|}{$55 \mathrm{mmHg}$} \\
\hline \multicolumn{3}{|c|}{ Right heart catheterization (RHC) } \\
\hline BSA & $1086 \mathrm{~m}^{2}$ & - \\
\hline MAP s/d/m & $94 / 65 / 75 \mathrm{mmHg}$ & \\
\hline RAP s/d/m & $7 / 2 / 5 \mathrm{mmHg}$ & Norm $0-5 \mathrm{mmHg}$ \\
\hline RVP s/d/m & $26 / 3 / 9 \mathrm{mmHg}$ & $\begin{array}{l}\text { Systolic: } 15-25 \mathrm{mmHg} \\
\text { Diastolic } 1-10 \mathrm{mmHg}\end{array}$ \\
\hline PAP s/d/m & $25 / 11 / 18 \mathrm{mmHg}$ & Systolic $15-25 \mathrm{mmHg}$; diastolic: $6-12 \mathrm{mmHg}$ \\
\hline mPAP & $14 \pm 3 \mathrm{mmHg}$ & Norm $<25 \mathrm{mmHg}$ \\
\hline PCW & $5 \mathrm{mmHg}$ & Norm $\leq 25 \mathrm{mmHg}$ \\
\hline $\mathrm{CO}-$ cardiac output & $8.98 \mathrm{l} / \mathrm{min} / \mathrm{m}^{2}$ & $>5 \mathrm{~L} / \mathrm{min}$ \\
\hline CI - cardiac index & $4.83 \mathrm{l} / \mathrm{min} / \mathrm{m}^{2}$ & $>2.41 / \mathrm{min} / \mathrm{m}^{2}$ \\
\hline PVR & $\begin{array}{l}116 \mathrm{dyn} \cdot \mathrm{sec}^{-1} \cdot \mathrm{cm}^{-5} \\
1.45 \mathrm{WU}\end{array}$ & Norm $<3 \mathrm{WU}\left(\leq 240 \mathrm{dyn} \cdot \mathrm{sec}^{-1} \cdot \mathrm{cm}^{-5}\right)$ \\
\hline PVRI & $\begin{array}{l}215 \mathrm{dyn} \cdot \mathrm{sec}^{-1} \cdot \mathrm{cm}^{-5} \mathrm{~m}^{2} \\
215 / 3=2.7 \mathrm{WU}\end{array}$ & PAH conformation $>6 \mathrm{WU}\left(>480 \mathrm{dyn} \cdot \mathrm{s}^{-1} \cdot \mathrm{cm}^{-5}\right)$ \\
\hline SVR & $\begin{array}{l}624 \mathrm{dyn} \cdot \mathrm{sec}^{-1} \cdot \mathrm{cm}^{-5} \\
=7.8 \mathrm{Wood} \text { units }\end{array}$ & Norm $8.8-20 \mathrm{WU}$ \\
\hline \multicolumn{3}{|l|}{ Spirometry } \\
\hline FEV1 & 2.2 litre & $84 \%$ norm \\
\hline FEV\%FVC & 84.29 & $105 \%$ norm \\
\hline DLCO kHb & $6.47 \mathrm{mmol} / \mathrm{min} / \mathrm{kPa}$ & $79 \% \mathrm{~N}$ \\
\hline 6MWT & 545 meters & $400-700$ meters \\
\hline
\end{tabular}

WBC - white bloodcount; HGB - hemoglobin; PLT - platelets; ESR - erythrocyte sedimentation rate; ALAT alanine transaminase; AST - aspartate aminotransferase; CK - creatine kinase; LDH - lactate dehydrogenase; ANA - 
antinuclear antibodies; EF - ejection fraction; TAPSE - tricuspid annular plane systolic excursion; RVSP - right ventricular systolic pressure; PAH: pulmonary arterial hypertension; BSA - body surface area; MAP s/d/m - mean arterial pressure: systolic, diastolic, medium; RAP s/d/m - right arterial pressure - systolic/ diastolic/medium; RVP right ventricular pressure - systolic/diastolic/medium; PAP: pulmonary arterial pressure - systolic/diastolic/medium; mPAP: mean pulmonary arterial pressure; PCW - pulmonary artery wedge pressure; CO - cardiac output; CI cardiac index; PVR: pulmonary vascular resistance; PVRI: pulmonary vascular resistance index; WU: Wood units; SVR: systemic vascular resistance

Table 1: Biochemical parameters and echocardiography measurements of analysed patient.

Besides, a ratio of PVR to SVR was low and ranged at 0.017 (the value PVR/SVR over $>0.75$ indicates significant pulmonary vascular disease). The amount of mPAP over $25 \mathrm{mmHg}$, elevated pulmonary vascular resistance over 3 Wood Units and decreased cardiac output are characteristic for PAH [3 Hoeper, 4 Galiè] [44, 45]. Fortunately, such changes were not present in this case. It has to be underlined that RHC plays a central role in identifying pulmonary hypertension disorders and is the diagnostic gold standard for pulmonary hypertension [51]. After an analysis of above mentioned data, pulmonary hypertension was excluded; however, the patients is under regular cardiologic control (Graph 1 and 2).

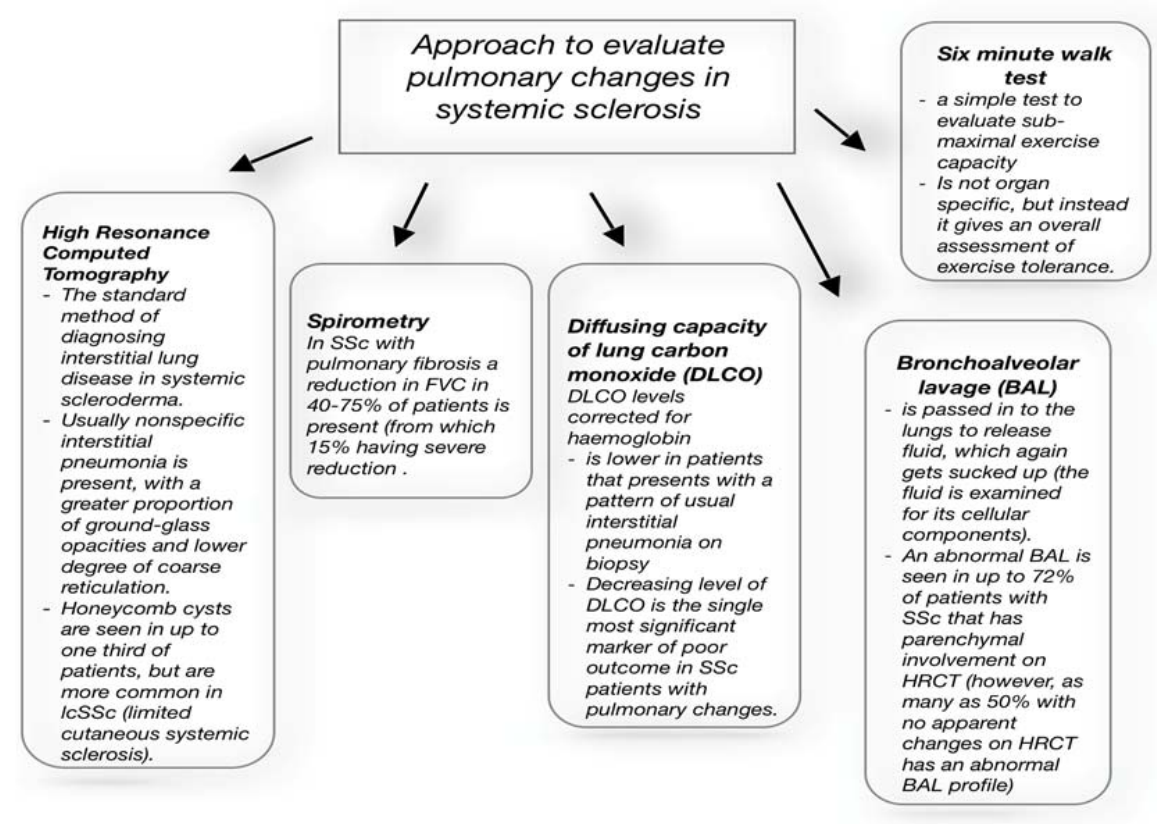

Graph 1: Methods of evaluating pulmonary involvement in SSc. 


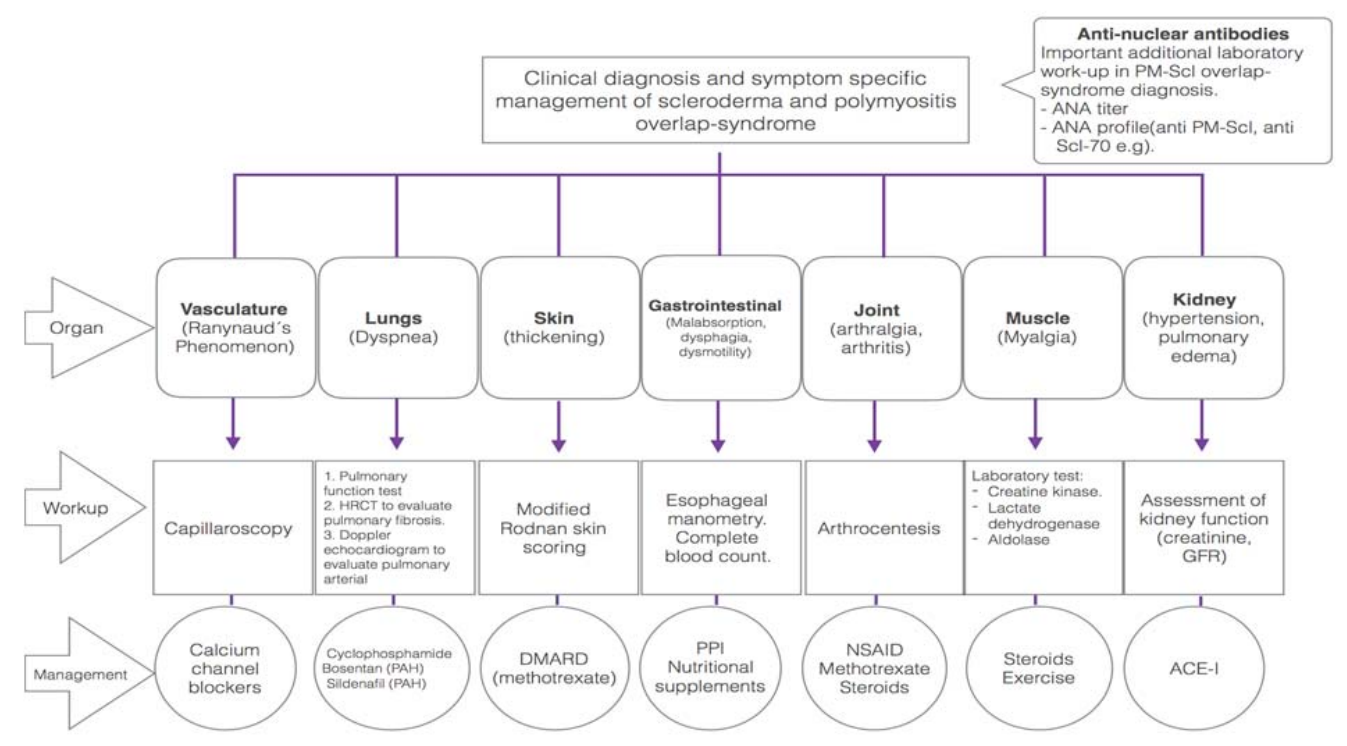

Graph 2: Organ specific approach for diagnosis and management of scleroderma and polymyositis overlapsyndrome.

\section{Prognosis and Treatment}

A prognostic algorithm has been suggested by Goh et al. that measures baseline PFT and HRCT, with the purpose of differentiating patients with extensive and limited disease. Patients with $>20 \%$ abnormalities on HRCT are considered to have extensive lung disease, while HRCT abnormalities $<20 \%$ are considered limited. If the HRCT is inconclusive, $\mathrm{FVC}<70 \%$ is regarded as extensive lung disease while $>70 \%$ is regarded as limited. Patients with extensive lung disease have been found to have a much higher mortality rate and more progressive lung deterioration [51]. The five-year survival rate for SSc-ILD is estimated to be $85 \%$, and early death is relatively uncommon [52]. In contrast to idiopathic interstitial pneumonia, survival rates does not differ between the pathological patterns of NSIP and UIP [30]. It is of great importance to recognize patients who are at risk to develop extensive SSc-ILD due to the fact that mortality rates are so much higher and treatment should be initiated as early as possible. Risk factors such as sex, race and antibodies such as anti-topoisomerase-1 which have been discussed previously has to be taken into account [53]. Besides, it has been suggested that patients with SSc-PM overlap-syndrome have an increased risk of mortality due to ILD and cardiac disease when compared to the single entities of either systemic sclerosis or polymyositis [54].

The patient was treated with cyclophosphamide (CYC) and low dose glucocorticosteroids, which is considered as the current mainstay of treatment in SSc-associated ILD. The effect of cyclophosphamide on forced vital capacity (FVC) in patients with SSc and ILD was significant but modest $[55,56]$ with an observed reduction of dyspnea but without effect on DLCO rates [57]. There is no treatment that can reverse lung fibrosis, therefore the viable treatment used in SSc-ILD is focused on the preceding inflammation that is thought to lead to fibrosis. In this case, 
fibrosis was present in HRCT and treatment with methylprednisolone and CYC (total dose $3600 \mathrm{mg}$ i.v.) were implemented. CYC is a drug that has shown good promise if implemented early.

In 2009 the Scleroderma Lung Study I demonstrated significant treatment-associated improvements in PFT when treated with CYC for 1 year [58]. Two double blinded [58, 59] and one unblinded [60] randomized controlled trials (RTC) has shown that patients treated with CYC has an increased FVC. However, the need for maintenance doses are apparent [59]. In 2015, the Scleroderma Lung Study II attempted to compare the effect of a 1-year trial of CYC vs a 2-year trial of mycophenolate mofetil (MMF) in a large double blind, RTC [61]. Both drugs were found to have good comparable effects on FVC after 1-year, and the Transition Dyspnea Index (TDI) and MRSS were improved in both treatment groups. However, the effect on TDI and MRSS was better in the group treated with CYC. On the other hand, fewer people withdrew from the MFF-group and leukopenia/thrombocytopenia were noted significantly less frequently in the MFF-group [61]. Corticosteroids are frequently used in SSc patients, usually in combination with other immunosuppressives e.g. CYC, like in this case. Pulses with corticosteroids used in association with CYC has shown promising results on PFT and HRCT [62, 63].

The implemented treatment in this case reduced dyspnea and weakness, and it stopped the progression of lung fibrosis. Improvement of skin changes were not observed, but the patient reported increased body mass after glucocorticosteroid use. The prevention of osteoporosis was achieved with regular supplementation of vitamin D and calcium, as well as with regular bone mineral density controls using densitometry.

\section{Conclusion}

The patient presented in this case report was initially diagnosed with systemic sclerosis on the basis of cutaneous and pulmonary findings in addition to the ANA-profile. However, on follow up the patient presented with muscle weakness and elevated values of aldolase, LDH and CK. The newly presented findings in the presence of PM-Scl antibodies lead to an updated diagnosis of scleromyositis overlap-syndrome. Early identification of an overlapsyndrome (like in this case) is important with regards to management and effectively slowing disease progression. Hence, comprehensive knowledge of disease progression and prognosis in both entities must be respected. The echocardiography in the patient suggested PAH, but RHC failed to confirm it. Regardless, the findings on ECHO requires further monitoring with addition of other cardiac function tests. Cardiac involvement is one of the most feared complications in scleromyositis overlap-syndrome and a leading cause of mortality. Likewise, early identification and management of interstitial lung disease in systemic sclerosis is crucial as it is considered the leading cause of poor disease progression and mortality in SSc. Early initiation of aggressive cyclophosphamide therapy as in the presented case is found to slow the progression of fibrosis, and is considered the "gold standard" mainstay treatment in ILD associated with SSc.

\section{Conflict of Interest}

The Authors declare that there are no conflicts of interest. 


\section{References}

1. Krieg T, Takehara K. Skin disease: a cardinal feature of systemic sclerosis. Rheumatology 48 (2009): iii14iiil8.

2. Schurawitzki H, Stiglbauer R, Graninger W, et al. Interstitial lung disease in progressive systemic sclerosis: high-resolution CT versus radiography. Radiology 176 (1990): 755-759.

3. Garin MC, Highland KB, Silver RM, et al. Limitations to the 6-minute walk test in interstitial lung disease and pulmonary hypertension in scleroderma.J Rheumatol 36 (2009): 330-336.

4. Walker UA, et al.Clinical risk assessment of organ manifestations in systemic sclerosis: a report from the EULAR Scleroderma Trials And Research group database Ann Rheum Dis 66 (2007): 754-763.

5. Reveille JD, Solomon DH. Evidence-based guidelines for the use of immunologic tests: Anticentromere, Scl-70, and nucleolar antibodies. Arthritis Care \& Research 49 (2007): 399-412.

6. Lóránd V, Czirják L, Minier T. Musculoskeletal involvement in systemic sclerosis. Presse Med 43 (2014): e315-328.

7. Ranque B, Authier FJ, Le-Guern V, et al. A descriptive and prognostic study of systemic sclerosisassociated myopathies. Ann Rheum Dis 68 (2009): 1474-1477.

8. Johnson SR, Glaman DD, Schentag CT, et al. Quality of life and functional status in systemic sclerosis compared to other rheumatic diseases. J Rheumatol 33 (2006): 1117-1122.

9. Randone SB, Guiducci S, Cerinic MM. Musculoskeletal involement in systemic sclerosis. Best Pract Res Clin Rheumatol 22 (2008): 339-350.

10. West SG, Killian PJ, Lawless OJ. Association of myositis and myocarditis in progressive systemic sclerosis. Arthritis Rheum 24 (1981): 662-668.

11. Boueiz A, Mathai SC, Hummers LK, et al. Cardiac complications of systemic sclerosis: recent progress in diagnosis. Curr Opin Rheumatol 22 (2010): 696-703.

12. Van Praet JT, et. al, Specific anti-nuclear antibodies in systemic sclerosis patients with and without skin involvement: an extended methodological approach. Rheumatology (Oxford) 50 (2011): 1302-1309.

13. Guldner HH, Szostecki C, Vosberg HP, et al. Scl 70 autoantibodies from scleroderma patients recognize a $95 \mathrm{kDa}$ protein identified as DNA topoisomerase I. Chromosoma 94 (1986): 132-138.

14. Spencer-Green G, Alter D, Gilbert Welch H. Test Performance in Systemic Sclerosis, Anti-Centromere and Anti-Scl-70 antibodies. The American Journal of Medicine 103 (1997): 242-248.

15. Patterson KA, et. al, Interpretation of an Extended Autoantibody Profile in a Well-Characterized Australian Systemic Sclerosis (Scleroderma) Cohort Using Principal Components Analysis. Arthritis Rheumatol 67 (2015): 3234-344.

16. Reimer G, Steen VD, Penning CA, et al. Correlates between autoantibodies to nucleolar antigens and clinical features in patients with systemic sclerosis (scleroderma). Arthritis Rheum 31 (1988): 525-532.

17. Martel C, et. al, Contribution of dot-blot assay to the diagnosis and management of myositis: a three-year practice at a university hospital centre.Clin Exp Rheumatol 34 (2016): 918-924.

18. Gunawardena H. The Clinical Features of Myositis-Associated Autoantibodies: a Review Clinical Reviews in Allergy \& Immunology 52 (2017): 45-57. 
19. Oddis CV, Okano Y, Rudert WA, et al. Serum autoantibody to the nucleolar antigen PM-Scl. Clinical and immunogenetic associations. Arthritis Rheum 35 (1992): 1211-1217.

20. Alderuccio F, Chan EK, Tan EM. Molecular characterization of an autoantigen of PM-Scl in the polymyositis/scleroderma overlap syndrome: a unique and complete human cDNA encoding an apparent 75-kD acidic protein of the nucleolar complex. J Exp Med 173 (1991): 941-952.

21. Blüthner M, Bautz FA. Cloning and characterization of the cDNA coding for a polymyositis-scleroderma overlap syndrome-related nucleolar 100-kD protein. J Exp Med 176 (1992): 973-980.

22. Hanke K, et al. Antibodies against PM/Scl-75 and PM/Scl-100 are independent markers for different subsets of systemic sclerosis patients. Arthritis Res Ther 11 (2009): R22.

23. Novel aspects of autoantibodies to the PM/Scl complex: Clinical, genetic and diagnostic insights.Mahler M, Raijmakers R. Autoimmun Rev 6 (2007): 432-437.

24. Avouac J, Fransen J, Walker U, et al. Preliminary criteria for the very early diagnosis of systemic sclerosis: results of a Delphi Consensus Study from EULAR Scleroderma Trials and Research Group. Ann Rheum Dis 70 (2011): 476-481.

25. Cutolo M, Sulli A, Secchi ME, et al. The contribution of capillaroscopy to the differential diagnosis of connective auto immune diseases. Best Pract Res Clin Rheum 21 (2007): 1093-1108.

26. Cutolo M, Sulli A, Secchi ME, et al. Nailfold capillaroscopy is useful for the diagnosis and follow-up of autoimmune rheumatic diseases. A future tool for the analysis of microvascular heart involvement? Rheumatology (Oxford) 45 (2006): iv43-46.

27. Ingegnoli F, Ardoino I, Boracchi P, et al. Nailfold capillaroscopy in systemic sclerosis: Data from the EULAR scleroderma trials and research (EUSTAR) database. Microvascular Res 89 (2013): 122-128.

28. Silver KC, Silver RM. Management of Systemic Sclerosis-Associated Interstitial Lung Disease (SSc-ILD). Rheumatic diseases clinics of North America 41 (2015): 439-457.

29. Solomon JJ, Olson AL, Fischer A, et al. Scleroderma lung disease. European Respiratory Review 22 (2013) 6-19.

30. Buch MH, et. al. Submaximal exercise testing in the assessment of interstitial lung disease secondary to systemic sclerosis: reproducibility and correlations of the 6-min walk test. Ann Rheum Dis 66 (2007): 169173.

31. Lopes AJ, Ferreira Ade S, Lima TR, et al. An explanatory model of functional exercise capacity in patients with systemic sclerosis: considerations for rehabilitation programs. J Phys Ther Sci 28 (2016): 569-575.

32. Machin DR, et al.Exercise-induced brachial artery blood flow and vascular function is impaired in systemic sclerosis. Am J Physiol Heart Circ Physiol 311 (2016): H1375-H1381.

33. Kovacs G, et al.Borderline pulmonary arterial pressure is associated with decreased exercise capacity in scleroderma. Am J Respir Crit Care Med 180 (2009): 881-886.

34. Lefère G, et al, Survival and Prognostic Factors in Systemic Sclerosis-Associated Pulmonary Hypertension: A Systematic Review and Meta-Analysis. Arthritis \& Rheumatology 65 (2013): 2412-2423.

35. Deuschle K, Weinert K, Becker MO, et al. Six-minute walk distance as a marker for disability and complaints in patients with systemic sclerosis.Clin Exp Rheumatol (2011): S53-S59. 
36. Impens AJ, Wangkaew S, Seibold JR. The 6-minute walk test in scleroderma-how measuring everything measures nothing. Rheumatology (Oxford) 47 (2008): v68-v69.

37. Enright PL. The six minut walk test. Respir Care 48 (2003): 783-785.

38. Bouros D, Wells AU, Nicholson AG, et al. Histopathologic subsets of fibrosing alveolitis in patients with systemic sclerosis and their relationship to outcome. Am J Respir Crit Care Med 165 (2002): 1581-1586.

39. Schurawitzki H, Stiglbauer R, Graninger W, et al. Interstitial lung disease in progressive systemic sclerosis: high-resolution CT versus radiography. Radiology 176 (1990): 755-759.

40. Khanna D, et al.Clinical Course of Lung Physiology in Patients with Scleroderma and Interstitial Lung Disease: Analysis of the Scleroderma Lung Study Placebo Group. Arthritis Rheum 63 (2011): 3078-3085.

41. Steen VD, Medsger TA. Changes in causes of death in systemic sclerosis, 1972-2002. Annals of the Rheumatic Diseases 66 (2007): 940-944.

42. Walker UA, Tyndall A, Czirják L, et al Clinical risk assessment of organ manifestations in systemic sclerosis: a report from the EULAR Scleroderma Trials And Research group database. Annals of the Rheumatic Diseases 66(2007): 754-763.

43. Scleroderma lung disease. Joshua J. Solomon, Amy L. Olson, Aryeh Fischer, Todd Bull, Kevin K. Brown, Ganesh Raghu. European Respiratory Review 22 (2013): 6-19.

44. Silver KC, Silver RM. Management of Systemic Sclerosis-Associated Interstitial Lung Disease (SSc-ILD). Rheumatic diseases clinics of North America 41 (2015): 439-457.

45. Demir N, Şahin A, Küçükşahin O, et al. Pulmonary arterial hypertension and systemic sclerosis relation: a single centre experience. Heart Lung Circ 23 (2014): 667-673.

46. Strollo D, Goldin J. Imaging Lung Disease in Systemic Sclerosis. Current Rheumatology Reports 12 (2010):156-161.

47. Hunninghake GW, Lynch DA, Galvin JR, et al. Radiologic findings are strongly associated with a pathologic diagnosis of usual interstitial pneumonia. Chest 124 (2003): 1215-1223.

48. Launay D, Remy-Jardin M, Michon-Pasturel U, et al. High resolution computed tomography in fibrosing alveolitis associated with systemic sclerosis. J Rheumatol 33 (2006): 1789-1801.

49. Meyer KC. Diagnosis and management of interstitial lung disease. Translational Respiratory Medicine 2 (2014): 4.

50. Meyer KC, Raghu G, Baughman RP, et al. An official American Thoracic Society clinical practice guideline: the clinical utility of bronchoalveolar lavage cellular analysis in interstitial lung disease. Am J Respir Crit Care Med 185 (2012): 1004-1014.

51. Goh NS, Desai SR, Veeraraghavan S, et al. Interstitial lung disease in systemic sclerosis: a simple staging system. Am J Respir Crit Care Med 177 (2008): 1248-1254.

52. Wells AU, Cullinan P, Hansell DM, et al. Fibrosing alveolitis associated with systemic sclerosis has a better prognosis than lone cryptogenic fibrosing alveolitis. Am J Respir Crit Care Med 149 (1994): 15831590.

53. Greidinger EL, Flaherty KT, White B, et al. African-American race and antibodies to topoisomerase I are associated with increased severity of scleroderma lung disease. Chest 114 (1998): 801-807. 
54. Bhansing KJ, Lammens M, Knaapen HK, et al. Scleroderma-polymyositis overlap syndrome versus idiopathic polymyositis and systemic sclerosis: a descriptive study on clinical features and myopathology. Arthritis Research \& Therapy 16 (2014): R111.

55. Tashkin DP, et al. Cyclophosphamide versus placebo in scleroderma lung disease. N Engl J Med 354 (2006): 2655-2666.

56. Hoyles RK, et al. A multicenter, prospective, randomized, double-blind, placebo-controlled trial of corticosteroids and intravenous cyclophosphamide followed by oral azathioprine for the treatment of pulmonary fibrosis in scleroderma. Arthritis \& Rheumatology 54 (2006): 3962-3970.

57. Poormoghim H, Moradi Lakeh M, Mohammadipour M, et al. Cyclophosphamide for scleroderma lung disease: a systematic review and meta-analysis. Rheumatol Int 32 (2012): 2431-2444.

58. Tashkin DP, Elashoff R, Clements PJ, et al. Effects of 1-year treatment with cyclophosphamide on outcomes at 2 years in scleroderma lung disease. Am J Respir Crit Care Med 76 (2007): 1026-1034.

59. Hoyles RK, Ellis RW, Wellsbury J, et al. A multicenter, prospective, randomized, double-blind, placebocontrolled trial of corticosteroids and intravenous cyclophosphamide followed by oral azathioprine for the treatment of pulmonary fibrosis in scleroderma. Arthritis Rheum 54 (2006): 3962-3970.

60. Nadashkevich O, Davis P, Fritzler M, et al. A randomized unblinded trial of cyclophosphamide versus azathioprine in the treatment of systemic sclerosis. Clin Rheum 25 (2006): 205-212.

61. Tashkin DP, Roth MD, Clements PJ. Sclerodema Lung Study II Investigators, et al. Mycophenolate mofetil versus oral cyclophosphamide in scleroderma-related interstitial lung disease (SLS II): a randomised controlled, double-blind, parallel group trial. Lancet Respir Med 4 (2016): 708-719.

62. Griffiths B, Miles S, Moss H, et al. Systemic sclerosis and interstitial lung disease: a pilot study using pulse intravenous methylprednisolone and cyclophosphamide to assess the effect on high resolution computed tomography scan and lung function. J Rheumatol 29 (2002): 2371-2378.

63. Yiannopoulos G, Pastromas V, Antonopoulos I, et al. Combination of intravenous pulses of cyclophosphamide and methylprednisolone in patients with systemic sclerosis and interstitial lung disease. Rheumatol Int 27 (2007): 357-361.

Citation: Bogna Grygiel-Górniak, Oscar Nicholas Godtfredsen, Gunnar Nyborg Eid, Nicholas Werczak, Mariusz Puszczewicz. Clinical Aspects of Overlap Syndrome - Case Report and Literature Review. Archives of Clinical and Biomedical Research 2 (2018): 117-131.

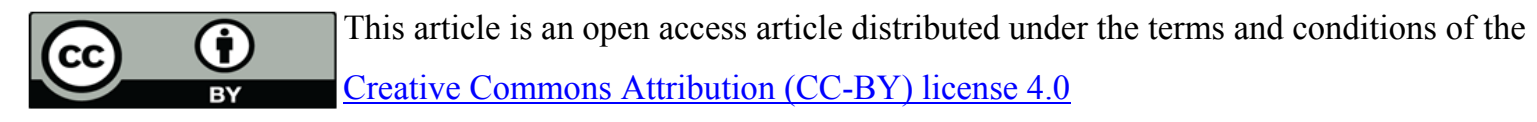

